\title{
Breast reconstruction using delayed pedicled transverse rectus abdominis muscle flap with supercharging: reports of three cases
}

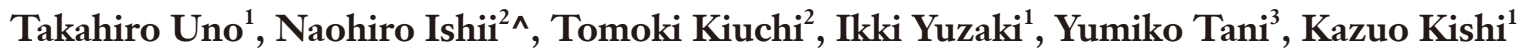 \\ ${ }^{1}$ Department of Plastic and Reconstructive Surgery, Keio University, Tokyo, Japan; ${ }^{2}$ Department of Plastic and Reconstructive Surgery, International \\ University of Health and Welfare Hospital, Tochigi, Japan; ${ }^{3}$ Department of Plastic and Reconstructive Surgery, Saiseikai Utsunomiya Hospital, \\ Utsunomiya City, Tochigi, Japan \\ Correspondence to: Naohiro Ishii, MD, PhD. Department of Plastic and Reconstructive Surgery, International University of Health and Welfare \\ Hospital, 537-3 Iguchi, Nasushiobara City, Tochigi 329-2763, Japan. Email: ishinao0916@gmail.com.
}

\begin{abstract}
Breast reconstruction using a pedicled transverse rectus abdominis muscle (TRAM) flap is a well-established surgical procedure. Although studies suggest that transplanting this flap using a delayed method reduces the risk of partial flap necrosis, challenges persist. Hence, we present three cases of breast reconstruction using a pedicled TRAM flap with both delaying and supercharging. Patient age, excised tissue volume for mastectomy, and follow-up period were as follows: Case 1, 58 years, $429 \mathrm{~cm}^{3}, 5$ months; Case 2, 35 years, $910 \mathrm{~cm}^{3}, 6$ months; and Case 3, 56 years, $489 \mathrm{~cm}^{3}, 4$ months. One patient (Case 2) required a large flap tissue volume to achieve breast symmetry, whereas the other two (Cases 1 and 3) had long, longitudinal scars from previous cesareans sections. In a delayed surgery, the flap was partially elevated with partial dissection and no ligation of the deep inferior epigastric artery and vein (DIEAV). An artificial dermis with a silicone membrane (Teldermis ${ }^{\circledR}$ ) was used to prevent adhesion of the rectus abdominal muscles and DIEAV to the surrounding tissue. Supercharging was performed by anastomosis between the ipsilateral DIEAV and internal thoracic AV. Flaps in zones I-III and in half of zone IV for Case 2, and zones I-III for Cases 1 and 3 , were transferred; all survived without infection. This method allowed the transferring of a larger tissue volume compared with the conventional pedicled TRAM flap-transfer method. Thus, it may be useful for patients who require larger tissue volume or high-risk patients.
\end{abstract}

Keywords: Breast reconstruction; transverse rectus abdominis muscle (TRAM); delay; supercharge; pedicled flap

Submitted May 20, 2021. Accepted for publication Jul 14, 2021.

doi: 10.21037 /gs-21-322

View this article at: https://dx.doi.org/10.21037/gs-21-322

\section{Introduction}

Breast reconstruction using a pedicled transverse rectus abdominis muscle (TRAM) flap may cause partial flap necrosis outside of zone I; hence, it is difficult to obtain a large tissue volume (1-3). The risk of flap necrosis decreases when blood circulation is improved using a delayed method, which divides the surgery into two stages (1-10). However, complete flap survival in zones I-III, especially in highrisk patients, remains challenging. To date, there are no studies that have reported a technique combining delaying with partial flap elevation, no ligation of the deep inferior epigastric artery and vein (DIEAV), and supercharging of the DIEAV.

This case report aims to present findings in using delayed pedicled TRAM flap with DIEAV supercharging for three patients, one requiring a large flap tissue volume to obtain breast symmetry, and the other two with longitudinal scars from a previous cesarean section. We present the following

^ ORCID: 0000-0001-8838-5344. 
cases in accordance with the CARE reporting checklist (available at https://dx.doi.org/10.21037/gs-21-322).

\section{Case presentation}

All procedures performed in studies involving human participants were in accordance with the ethical standards of the institutional and/or national research committee(s) and with the Helsinki Declaration (as revised in 2013). Written informed consent was obtained from all the patients prior to their participation in the study.

\section{Case 1}

The patient was a 58 -year-old female with a body mass index (BMI) of $25.1 \mathrm{~kg} / \mathrm{m}^{2}$. She had no history of radiotherapy, smoking, or diabetes and underwent tissue expander insertion after simple mastectomy (Figure 1A). Since the excised tissue volume for the right mastectomy was $429 \mathrm{~cm}^{3}$, we included all zones I-III for reconstruction. All surgeries were performed under general anesthesia. The contralateral pedicled TRAM flap, including the rectus abdominis muscle and ipsilateral DIEAV, was elevated and transplanted to the chest after a delay of 4 days by supercharging. Following flap elevation, the rectus abdominis muscle surrounding the pedicle and ipsilateral DIEAV within the skin incision was dissected. The ipsilateral DIEAV was preserved without ligation. Zone I was detached and elevated from the rectus abdominis muscle attached to the upper half of the perforator region, and zones II-IV were separated from the anterior rectus abdominis sheath by making a long incision of the skin island as possible. Since the cesarean scar under the umbilicus occupied approximately half the vertical width of the skin island, no skin incision was made on the caudal side of zone II and zone IV was excluded (Figure 1B). The adhesion of the rectus abdominal muscle and DIEAV to the surrounding tissue was prevented using an artificial dermis with a silicone membrane (Teldermis ${ }^{\circledR}$ ) (Figure 1C), and the flap was sutured back to its original position.

After the delay, the rectus abdominis muscle and DIEAV were easily detached, with no adherence to the surrounding tissue, and additional dissection of the DIEAV to the external iliac artery and vein could be performed smoothly (Figure 1D). After skin incision and DIEAV cutting, a moderate insufficiency of blood circulation on the caudal side of zone II was noted. However, the circulation was improved by supercharging. The flap was placed vertically so that zone II was facing the cranial side, and supercharging was performed in an end-to-end fashion between the ipsilateral DIEAV and internal thoracic AV (Figure $1 E$ ) since the ipsilateral DIEAV can barely reach the thoracodorsal AV but can easily turn and reach the internal thoracic AV as reported $(11,12)$. During flap transfer, ischemia persisted for 87 minutes. We created a skin island to include zone I. The rest of the flap was de-epithelialized and implanted under the skin.

All marginal flap bleeding was good. No vascular anastomosis was performed perioperatively, and there was no flap necrosis, infection, fat-hardening, and fatlysis after elevation and transfer (Figure $1 F$ ). The follow-up period was 5 months.

\section{Case 2}

The patient was a 35-year-old female with a BMI of $26.0 \mathrm{~kg} / \mathrm{m}^{2}$. She had no history of radiotherapy, smoking, or diabetes and underwent tissue expander insertion after simple mastectomy (Figure 2A). The excised tissue volume for the right mastectomy was $910 \mathrm{~cm}^{3}$; hence, we included all of zones I-III and half of zone IV for the reconstruction. All surgeries were performed under general anesthesia. The contralateral pedicled TRAM flap, including the rectus abdominis muscle and ipsilateral DIEAV, was elevated and transplanted to the chest after a delay of 4 days, using supercharging. Following the flap elevation, the rectus abdominis muscle surrounding the pedicle and ipsilateral DIEAV within the skin incision was dissected. The ipsilateral DIEAV was preserved without ligation. Zone I was detached and elevated from the rectus abdominis muscle attached to the upper half of the perforator region, and zones II-IV were separated from the anterior rectus abdominis sheath by making a long incision of the skin island as possible. To maintain blood circulation in zone IV, no skin incision was made on its lateral and caudal sides, and the superficial inferior epigastric vein (SIEV) on the caudal side of zone II was identified and preserved (Figure 2B). Likewise, Teldermis ${ }^{\circledR}$ was subsequently placed, and the flap was sutured back to its original position.

After the delay, the rectus abdominis muscle and DIEAV could be easily detached, with no adherence to the surrounding tissue, and additional dissection of DIEAV to the external iliac artery and vein could be performed smoothly. After skin incision and DIEAV cutting, moderate impairment of blood circulation was seen in half of zone IV, which improved by supercharging. Additional anastomosis was not required since drainage from the SIEV on the 

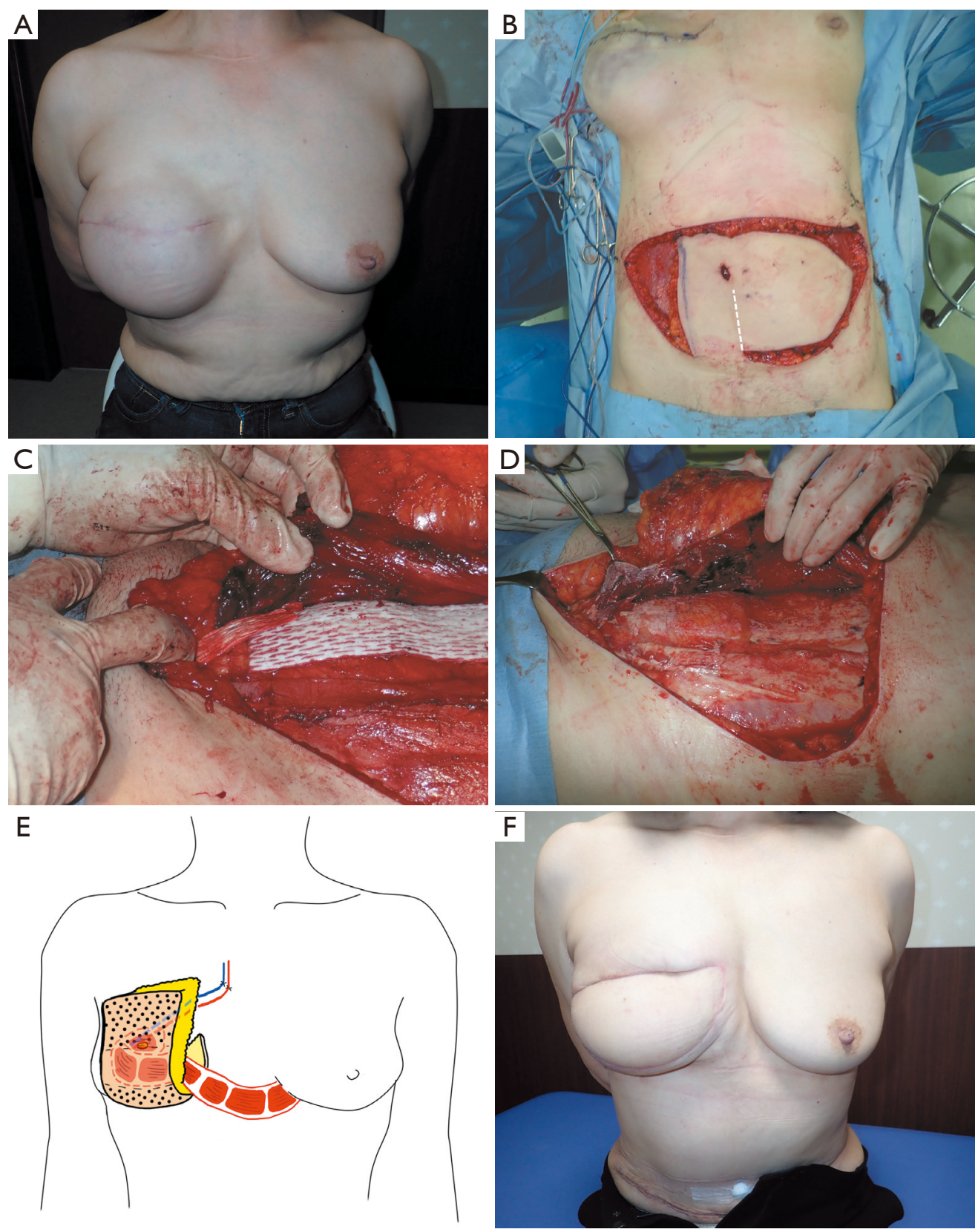

Figure 1 Sequential images of case 1 with a cesarean scar and undergoing flap transfer of all zones I-III. (A) Preoperative view after full expansion. (B) Skin incision was made with the exclusion of the caudal side of zone II, considering the longitudinal scar from abdominal surgery. The ipsilateral DIEAV were dissected within the skin incision and preserved without ligation. The white broken line indicates longitudinal scars from the cesarean section. (C) An artificial dermis with a silicon membrane was placed between the DIEAV and rectus abdominis muscle. (D) The rectus abdominis muscle and DIEAV were easily detached from the surrounding tissue. (E) Flap arrangement. The ipsilateral DIEAV turning and reaching internal thoracic artery and vein for supercharging. Dot area: denuded areas are shown; the cranial denuded area included zone II [+ partial zone IV (case 2)], and the caudal denuded area included partial zone I and zone III. (F) Appearance 3 months after flap transfer. Revision surgery is planned. 

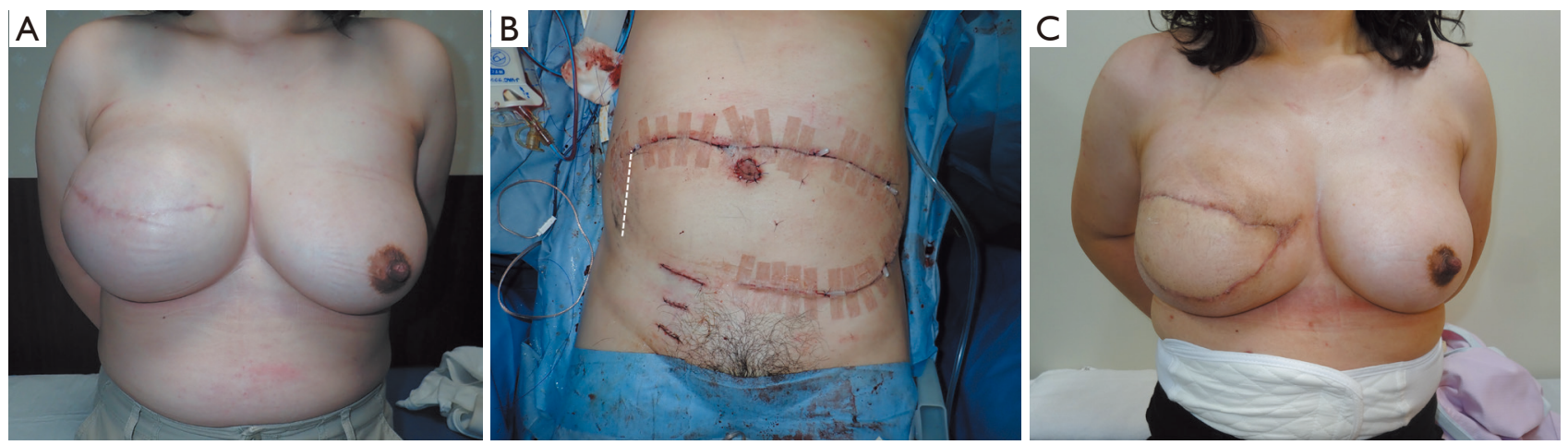

Figure 2 Sequential images of case 2 with undergoing flap transfer of all zones I-III and half of zone IV. (A) Preoperative view after full expansion. (B) A skin incision was made excluding the lateral and caudal side of zone $\mathrm{IV}$, and the superficial inferior epigastric vein was preserved in preparation for an additional anastomosis in flap-transfer. The ipsilateral DIEAV were dissected within the skin incision and preserved without ligation. White broken line indicates the skin incision at the midpoint of zone IV in flap-transfer. (C) Appearance 3 months after flap-transfer. Revision surgery is planned.

caudal side of zone II was not observed. The flap was placed vertically so that zone II was facing the cranial side, and supercharging was performed in an end-to-end fashion between the ipsilateral DIEAV and internal thoracic AV. During flap transfer, ischemia persisted for 95 minutes. We created the skin island to include zone I. The rest of the flap was de-epithelialized and implanted under the skin.

All marginal flap bleeding was good. No vascular anastomosis was performed perioperatively, and there was no flap necrosis, infection, fat-hardening, and fatlysis after elevation and transfer (Figure 2C). The follow-up period was 6 months.

\section{Case 3}

The patient was a 56-year-old female with a BMI of $18.6 \mathrm{~kg} / \mathrm{m}^{2}$. She had no history of radiotherapy, smoking, or diabetes and underwent tissue expander insertion after simple mastectomy (Figure $3 A$ ). As the excised tissue volume for the right mastectomy was $489 \mathrm{~cm}^{3}$, zones I-III were included for the reconstruction. All surgeries were performed under general anesthesia. The contralateral pedicled TRAM flap, including the rectus abdominis muscle and ipsilateral DIEAV, was elevated and transplanted to the chest after a delay of 4 days by supercharging. As the cesarean scar was similar to that of the patient in case 1 , the same procedure as in case 1 was performed, and a similar postoperative course was noted (Figure 3B).

After the delay, the rectus abdominis muscle and
DIEAV were easily separated from the surrounding tissue. Moreover, additional dissection of the DIEAV was performed smoothly. Furthermore, flap blood circulation was enhanced by supercharging. During flap transfer, ischemia persisted for 82 minutes. In addition, the circumvertical reduction mammoplasty of the contralateral breast was performed.

All marginal flap bleeding was good. No vascular anastomosis was performed perioperatively, and there was no flap necrosis, infection, fat-hardening, and fatlysis after elevation and transfer (Figure 3C). The follow-up period was 4 months.

\section{Discussion}

There are several methods for performing delayed pedicled TRAM flaps including partial elevation of pedicled TRAM flap with $(1,5,6,8)$ or without (2) DIEAV ligation and no flap elevation with DIEAV ligation by embolization using interventional radiology $(4,7)$ or under direct vision (3). The vascular territory of pedicled TRAM flap is zone I and partial zones II and III, since this flap is vascularized by the superior epigastric artery and vein (SEAV) through the choke vessels between the SEAV and DIEAV, including the main perforators to the TRAM flap (13-15). Surgical delay enhances the pedicled TRAM flap's vascularity to dilate the choked vessels of zones I-II and zones I-III; however, achieving complete flap survival in zones I-III remains difficult (1-8). Supercharging of the ipsilateral DIEAV can 

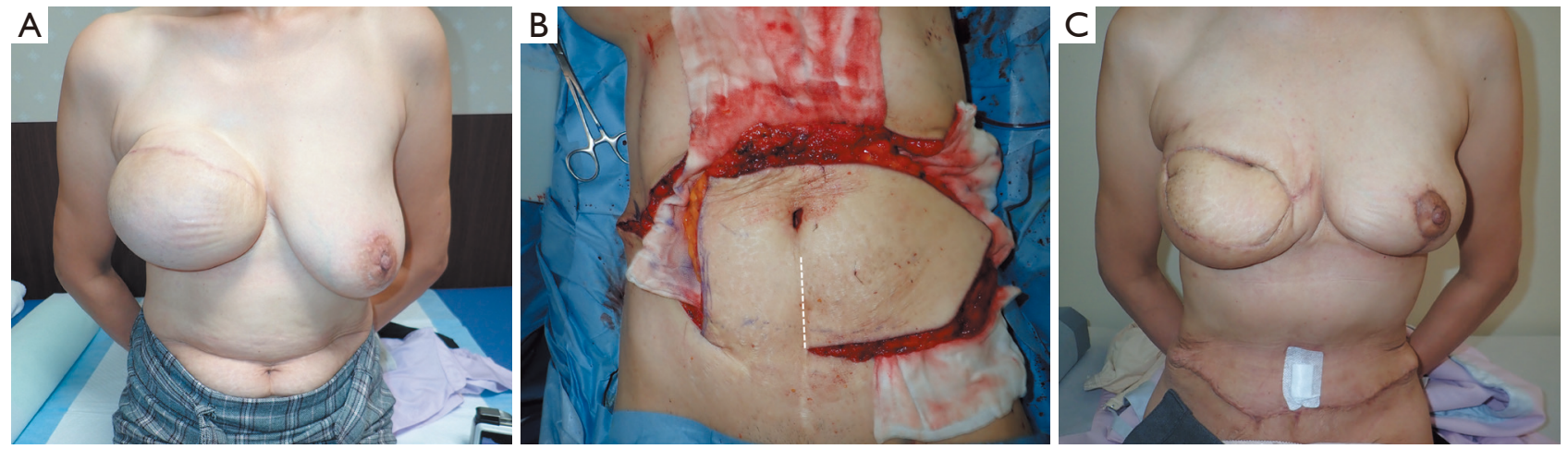

Figure 3 Sequential images of case 3 with a cesarean scar and undergoing flap transfer of all zones I-III. (A) Preoperative view after full expansion. (B) Skin incision was made, excluding the caudal side of zone II, considering the longitudinal scar from abdominal surgery. The ipsilateral DIEAV were dissected within the skin incision and preserved without ligation. White broken line indicates longitudinal scars from the cesarean section. (C) Appearance 3 months after flap-transfer. Revision surgery is planned.

provide a large vascular territory of this flap involving zones I-III in many cases; however, even with this technique, partial flap necrosis of zone II in cases with cesarean scars and flap necrosis of zone IV may occur $(11,12)$.

Two studies involving human subjects have reported delaying the pedicled TRAM flaps coupled with DIEAV supercharging $(9,10)$. One revealed that delay without flap elevation coupled with ipsilateral DIEAV ligation following subsequent supercharging of the ipsilateral DIEAV was a viable option involving the troublesome removal of the thrombus during flap transfer. However, it was not clear how a significant enhancement of vascularity in this flap was provided (9). Furthermore, the other mentioned that delay with the partial flap elevation coupled with ipsilateral DIEAV ligation following subsequent contralateral DIEAV supercharging could provide complete flap survival in zones I-IV due to dilation of the choked vessels of zones I-III and zones II-IV (10). However, additional harvesting of the contralateral DIEAV might be invasive.

One study involving rat subjects reported delaying the pedicled TRAM flaps coupled with DIEAV supercharging (16). This article reported a 2 -week delay of the inferior pedicled TRAM flaps coupled with supercharging of the ipsilateral cranial epigastric vein; the flap survival area was compared between group A [delay (-), supercharging (-)], group B [delay (-), supercharging (+)], group C [delay $(+)$, supercharging $(-)$ ], and group D [delay $(+)$, supercharging $(+)]$. The delay technique involved the ligation of the cranial epigastric artery with a small skin incision and no flap elevation. Supercharging meant the preservation of the cranial epigastric vein, including no vascular anastomosis. The results showed that group D had the largest flap survival area, followed by group C, group B, and group A. Consequently, this study suggested that the flap survival area can be maximized by delaying the pedicled TRAM flaps coupled with supercharging.

The flap elevation procedure is shown in Figure 4. Our methods included delay with partial flap elevation without ipsilateral DIEAV ligation following subsequent supercharging of the ipsilateral DIEAV. Flap survival was achieved up to half of zone IV in Case 2 since our method may enhance the vascularity of zone II in the horizontal direction to dilate the choked vessels of zones II-IV. Furthermore, even in Cases 1 and 3 with cesarean scars, complete survival of zone II was possible since our method may enhance the vascularity of zone II in the vertical direction to dilate the choked vessels of zones I-II.

The DIEV diameter in the conventional supercharged pedicled TRAM flap transfer is clinically known to be approximately $3 \mathrm{~mm}$ at the site flowing into the external iliac vein. In these three cases, however, the vascular diameters were smaller (2.2 mm, Case 1; $2.2 \mathrm{~mm}$, Case 2; and $1.8 \mathrm{~mm}$, Case 3). This suggests that the delay method may increase the drainage of the superior epigastric vein.

Our technique is novel in several aspects. First is the delayed method before flap transfer with supercharging of the ipsilateral DIEAV, which involves partial flap elevation with partial dissection and no ligation of the DIEAV. If subsequent supercharging with the ipsilateral DIEAV is planned, dissection of the DIEAV should be 


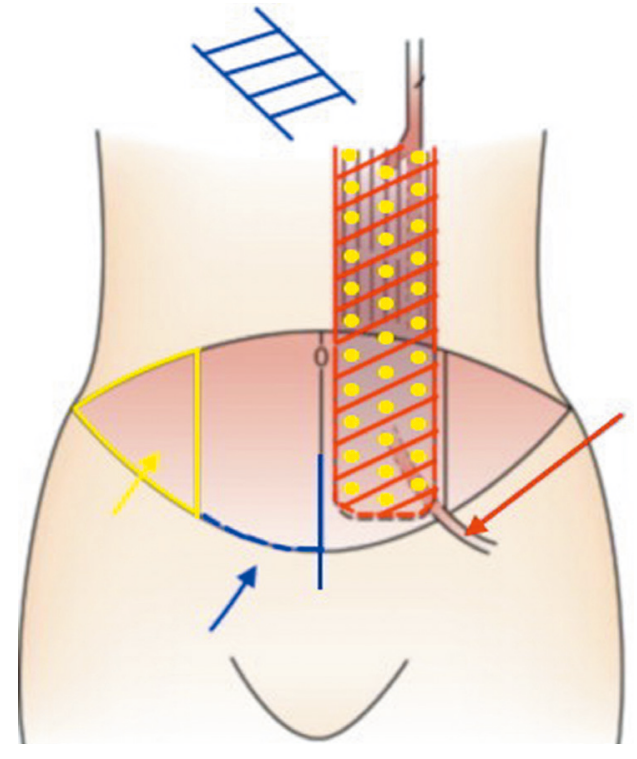

Figure 4 Schematic diagram of our flap elevation procedure. Red ladder: the rectus abdominis muscle, including the feeding blood vessels, should be completely dissected, except for those on the caudal side. Blue ladder: subcutaneous tunnel that should be created. Red arrow: DIEAV. Dissection should be performed within the skin incision. Blue arrow: longitudinal scar after abdominal surgery. If a longitudinal scar after abdominal surgery exists, incision should not be made on the caudal side of zone II. Yellow arrow: zone IV of the TRAM flap. Unused parts can be cut off. If this zone is used, the caudal side should not be cut. Yellow dots: area where silicone sheet should be placed. Feeding vessels and tissue containing the feeding vessels should be prevented from adhering to the surrounding tissue.

performed within the skin incision not to be buried in the surrounding tissue in flap transfer. Avoiding scar and longterm thrombus formation of the proximal DIEAV at the external iliac arteriovenous inflow site may reduce the risk of thrombus formation in vascular anastomosis. Therefore, the DIEAV should not be dissected along the entire length and should not be ligated during flap elevation.

Second is the use of silicone sheets in delayed surgery to prevent feeding vessels and tissue containing the feeding vessels from adhering to the surrounding tissue. Laying silicone sheets under zones III and IV to avoid revascularization from the rectus abdominis fascia has been reported only once (10). Since adhesion was observed in the areas where Teldermis ${ }^{\circledR}$ was not placed, adhesion of the rectus abdominis muscle, including the vascular pedicle and DIEAV, should be prevented using a silicone sheet.
Third is a short, delaying period. In many previous studies regarding delaying pedicled TRAM flaps, flap blood circulation enhancement was achieved with a delay of 1-2 weeks (1-9). With only a 4-day delay in our technique, flap blood circulation of zone II was enhanced as described above, thereby proving our procedure's effectiveness. The reason for this result may be that these articles reported that flap vascularity can be enhanced in the early postoperative period as the neo-vascularization and dilation of choke vessels are promoted $(17,18)$. Additionally, subsequent dissection of DIEAV in flap transfer may be difficult due to scarring if the delaying period in our technique is more than 7 days, similar to what we experienced in another similar case of delayed deep inferior epigastric artery perforator (DIEP) flap that had a seven-day delay.

The bipedicled free TRAM flap may be often used for high-risk patients or those requiring larger tissue volumes. This flap is generally used in one-stage surgery and requires vascular anastomosis of two pairs of vessels at two different sites. The rate of total flap loss using free TRAM flap is low, since this rate using free TRAM flap without DIEP flap is lower than $3.5 \%$ and this rate using DIEP flap is lower than $5.0 \%$ (19-21). Our delayed pedicled TRAM flap with supercharging is used in two-stage surgery with some additional costs; however, each operation is not timeconsuming, and vascular anastomosis of only one pair of vessels is required. Surgical delay and supercharging have already been well established in the literature that their use would be ways to increase the size of a pedicled TRAM flap (1-12). Furthermore, flap loss may not be so large even in cases of vascular anastomosis failure. All patients in this study would not like to undergo an operation that includes the possibility of total or heavily partial flap necrosis due to the obstruction of anastomosed vessels. Our technique may be a useful option if a bipedicled TRAM flap is unavailable for some reasons. Abdominal weakness, bulge, or hernia did not occur in all patients; however, a careful fascia closure considered to be used as a mesh for strengthening the abdominal wall depending on the situation should be performed to avoid these abdominal postoperative complications.

Since our method was employed in only three cases, more studies including more patients are warranted to further investigate the effectiveness and proper period of delaying and collect objective data on the flap survival area, which is evaluated by intraoperative indocyanine green angiography or ex vivo angiography. This method allowed the transfer of a larger tissue volume compared with the 
conventional pedicled TRAM flap-transfer method. Thus, it may be useful for patients who require larger tissue volume or those at high risk.

\section{Acknowledgments}

Funding: None.

\section{Footnote}

Reporting Checklist: The authors have completed the CARE reporting checklist. Available at https://dx.doi. org/10.21037/gs-21-322

Peer Review File: Available at https://dx.doi.org/10.21037/ gs-21-322

Conflicts of Interest: All authors have completed the ICMJE uniform disclosure form (available at https://dx.doi. org/10.21037/gs-21-322). The authors have no conflicts of interest to declare.

Ethical Statement: The authors are accountable for all aspects of the work in ensuring that questions related to the accuracy or integrity of any part of the work are appropriately investigated and resolved. All procedures performed in studies involving human participants were in accordance with the ethical standards of the institutional and/or national research committee(s) and with the Helsinki Declaration (as revised in 2013). Written informed consent was obtained from all the patients prior to their participation in the study.

Open Access Statement: This is an Open Access article distributed in accordance with the Creative Commons Attribution-NonCommercial-NoDerivs 4.0 International License (CC BY-NC-ND 4.0), which permits the noncommercial replication and distribution of the article with the strict proviso that no changes or edits are made and the original work is properly cited (including links to both the formal publication through the relevant DOI and the license). See: https://creativecommons.org/licenses/by-nc-nd/4.0/.

\section{References}

1. Khater A, Hamed E, Roshdy S, et al. A New Concept of Interval TRAM for Immediate Breast Reconstruction in Obese Women. Indian J Surg Oncol 2019;10:280-5.
2. Towpik E, Mazur S, Witwicki T, et al. Elevating the island: the simplest method of delaying the TRAM flap. Ann Plast Surg 2000;45:240-3.

3. Restifo RJ, Ahmed SS, Rosser J, et al. TRAM flap perforator ligation and the delay phenomenon: development of an endoscopic/laparoscopic delay procedure. Plast Reconstr Surg 1998;101:1503-11.

4. Aboutanos SZ, Spinos E, Blanchet NP. Angiographic delay: a viable alternative to surgical delay. Ann Plast Surg 2012;68:562-7.

5. O'Shaughnessy KD, Mustoe TA. The surgical TRAM flap delay: reliability of zone III using a simplified technique under local anesthesia. Plast Reconstr Surg 2008;122:1627-30.

6. Erdmann D, Sundin BM, Moquin KJ, et al. Delay in unipedicled TRAM flap reconstruction of the breast: a review of 76 consecutive cases. Plast Reconstr Surg 2002;110:762-7.

7. Scheufler O, Andresen R, Kirsch A, et al. Clinical results of TRAM flap delay by selective embolization of the deep inferior epigastric arteries. Plast Reconstr Surg 2000;105:1320-9.

8. Codner MA, Bostwick J 3rd, Nahai F, et al. TRAM flap vascular delay for high-risk breast reconstruction. Plast Reconstr Surg 1995;96:1615-22.

9. Vosburg RW, White MJ, Heckler FR. Supercharging of delayed pedicled transverse rectus abdominis myocutaneous flaps, is it a viable option? Microsurgery 2015;35:204-6.

10. Kajikawa A, Ueda K, Tateshita T, et al. Breast reconstruction using tissue expander and TRAM flap with vascular enhancement procedures. J Plast Reconstr Aesthet Surg 2009;62:1148-53.

11. Wu LC, Iteld L, Song DH. Supercharging the transverse rectus abdominis musculocutaneous flap: breast reconstruction for the overweight and obese population. Ann Plast Surg 2008;60:609-13.

12. Marck KW, van der Biezen JJ, Dol JA. Internal mammary artery and vein supercharge in TRAM flap breast reconstruction. Microsurgery 1996;17:371-4.

13. Boyd JB, Taylor GI, Corlett R. The vascular territories of the superior epigastric and the deep inferior epigastric systems. Plast Reconstr Surg 1984;73:1-16.

14. Taylor GI, Palmer JH. The vascular territories (angiosomes) of the body: experimental study and clinical applications. Br J Plast Surg 1987;40:113-41.

15. Moon HK, Taylor GI. The vascular anatomy of rectus abdominis musculocutaneous flaps based on the 
deep superior epigastric system. Plast Reconstr Surg 1988;82:815-32.

16. Sano K, Hallock GG, Rice DC. Venous "supercharging" augments survival of the delayed rat TRAM flap. Ann Plast Surg 2003;51:398-402.

17. Ichioka S, Minh TC, Shibata M, et al. In vivo model for visualizing flap microcirculation of ischemia-reperfusion. Microsurgery 2002;22:304-10.

18. Ishii N, Kiuchi T, Uno T, et al. Time-dependent changes in the circulation after the elevation of reverse flow sural flap. Microsurgery 2020;40:87-8.

19. Nahabedian MY, Momen B, Galdino G, et al. Breast

Cite this article as: Uno T, Ishii N, Kiuchi T, Yuzaki I, Tani Y, Kishi K. Breast reconstruction using delayed pedicled transverse rectus abdominis muscle flap with supercharging: reports of three cases. Gland Surg 2021;10(8):2577-2584. doi: 10.21037/gs21-322
Reconstruction with the free TRAM or DIEP flap: patient selection, choice of flap, and outcome. Plast Reconstr Surg 2002;110:466-75; discussion 476-7.

20. Nahabedian MY, Tsangaris T, Momen B. Breast reconstruction with the DIEP flap or the muscle-sparing (MS-2) free TRAM flap: is there a difference? Plast Reconstr Surg 2005;115:436-44; discussion 445-6.

21. Jeong W, Lee S, Kim J. Meta-analysis of flap perfusion and donor site complications for breast reconstruction using pedicled versus free TRAM and DIEP flaps. Breast 2018;38:45-51. 